\title{
Where is your head? Perception of relative position of the head on a wielded object
}

\author{
Jeffrey B. Wagman ${ }^{1} \cdot$ Takahiro Higuchi $^{2}$
}

Published online: 14 March 2019

(C) The Psychonomic Society, Inc. 2019

\begin{abstract}
Perception of exteroceptive properties (e.g., object length) by effortful or dynamic touch is both task-specific and anatomically independent. We investigate whether task-specificity and anatomical independence generalize to perception of proexteroceptive properties of the person-object system (i.e., relative position of the body on a wielded object). Moreover, we do so when objects are wielded by a body part that is unlikely to be well practiced in such tasks - the head. Experiment 1 found that participants can perceive the relative location of the head on a wielded object and that such perception is likely supported by task-specific sensitivity to an invariant mechanical stimulation pattern - rotational inertia. Experiment 2 found that participants have at least some ability to differentiate between this property and a related exteroceptive property (i.e., partial length of a wielded object extending to one side of the head). The results are discussed in terms of information for perception by effortful touch and a description of the haptic system as a biotensegrity structure.
\end{abstract}

Keywords Haptics $\cdot$ Perception and action $\cdot$ Touch

Manipulating the body and/or objects attached to the body by means of muscular effort engages the haptic subsystem known as dynamic or effortful touch (Gibson, 1966). Decades of research on perception by dynamic or effortful touch have shown that when wielding an occluded object, people can perceive many different geometric and functional properties of the person-object system including properties of (1) the object itself (exteroceptive properties), (2) the body itself (proprioceptive properties), (3) the object relative to the body (exproprioceptive properties), and (4) the body relative to the object (proexteroceptive properties; Carello \& Turvey, 2015; cf. Lee, 1978; Shaw, 2001).

Perception by dynamic touch exhibits marked flexibility in two important ways. First, as intimated above, it exhibits flexibility in the variety of properties that can be perceived both within (and across) bouts of wielding. Many different

Jeffrey B. Wagman

jeffreywagman@illinoisstate.edu; https://about.illinoisstate.edu/ jbwagma/Pages/default.aspx

1 Department of Psychology, Illinois State University, Campus Box 4620, Normal, IL 61790, USA

2 Department of Health Promotion Sciences, Tokyo Metropolitan University, Tokyo, Japan properties of (components of) a given person-object system can be perceived when an object is wielded in a particular manner, with a particular effector, about a particular joint. For example, when freely wielding an object with the hand about the wrist, people can perceive the length, width, shape, orientation, and utility of that object for performing a given task, among other properties (see Carello \& Turvey, 2015, 2017, for reviews).

Second, perception by dynamic touch exhibits flexibility in the means by which a given property of the person-object system can be perceived. A specific property of the personobject system can be perceived when a given object is wielded in different ways, by different effectors, about different joints. For example, people can perceive the length of an object when it is freely wielded or held with minimal movement (Burton \& Turvey, 1990; Carello Fitzpatrick, Domaniewicz, Chan, \& Turvey, 1992); when it is wielded with the hand about the wrist, the elbow, or the shoulder (Pagano, Fitzpatrick, \& Turvey, 1993); or when it is wielded by the hand, foot, torso, or head (Hajnal, Fonseca, Harrison, Kinsella-Shaw, \& Carello, 2007a; Palatinus, Carello, \& Turvey, 2011; Wagman, Langley, \& Higuchi, 2017).

Flexibility in both the variety of properties that can be perceived and in the variety of means by which such properties can be perceived is grounded in sensitivity to the rotational inertia $(I)$ 
of the person-object system. I quantifies the resistance of an object to rotational acceleration about a rotation point. Consequently, it describes the scaling and directing of the muscular forces required to control the object (or person-object system). Crucially, I lawfully couples wielding forces to wielding movements and is invariant across transformations including wielding movements and trajectories, points of rotation, grasp types and locations, and anatomical components.

Moreover, perception of particular exteroceptive, proprioceptive, exproprioceptive, and proexteroceptive properties is lawfully grounded in sensitivity to particular components of $I$. Perception of exteroceptive properties (e.g., length, width, shape) is primarily supported by sensitivity to the moments of inertia (e.g., $I_{x \mathrm{x}}, I_{\mathrm{yy}}, I_{\mathrm{zz}}$ ), which quantify rotational inertia with respect to the three orthogonal axes of rotation $(x, y$, and $z$, respectively). The moments of inertia reflect the symmetrical mass distribution of the person-object system about these axes. Accordingly, perception of the length and width of a wielded object are primarily supported by detection of $I_{\mathrm{xx}}$ and $I_{z z}$, respectively (Fitzpatrick, Carello, \& Turvey, 1994; Turvey, Burton, Amazeen, Butwill, \& Carello, 1998). However, perception of exproprioceptive properties (e.g., orientation of the wielded object relative to the body) and proexteroceptive properties (e.g., relative location of a body part on the wielded object) is primarily supported by sensitivity to the products of inertia (e.g., $I_{\mathrm{xy}}, I_{\mathrm{xz}}, I_{\mathrm{yz}}$ ), which quantify resistance to rotation about axes perpendicular to the $x, y$, and $z$ axes. The products of inertia reflect the asymmetrical mass distribution about these axes. Accordingly, perception of the location of the hand relative to a wielded object is supported by detection of $I_{\mathrm{yz}}$ (Pagano, Carello, \& Turvey, 1996; Pagano, Kinsella-Shaw, Cassidy, \& Turvey, 1994).

Overall, studies such as the ones cited above have shown that perception by effortful touch is grounded in the intentional detection of task-specific components of an invariant stimulation pattern $(I)$. The invariant nature of $I$ is highlighted not only by the ability to perceive a given property within and across bouts of wielding but also by the ability to perceive a given property by different means of wielding-including cases in which an object is wielded by disparate anatomical components. Studies investigating this latter ability have shown that people can perceive exteroceptive properties of a wielded object such as whole or partial length when an object is wielded by the hand, the torso, the foot, and even the head (Hajnal, Fonseca, Kinsella-Shaw, et al., 2007b; Wagman et al., 2017). Moreover, they can differentiate between two different (but related) exteroceptive properties (e.g., whole length vs. partial length, probe length vs. distance of surface probed) under such circumstances (Hajnal, Fonseca, Kinsella-Shaw, et al., 2007a; Palatinus et al., 2011; Wagman et al., 2017; Wagman \& Mason, 2019).

Together, the specificity of perception by dynamic touch to $I$ and the specificity of $I$ to properties of the person-object system entail that perception by dynamic touch is both taskspecific and anatomically independent. Previous research has found evidence of both such attributes in perception of exteroceptive properties of wielded objects. In two experiments, we investigate whether task-specificity and anatomical independence generalize to perception of proexteroceptive properties of the person-object system (i.e., relative position of the body on a wielded object). Moreover, we investigate such generalization under conditions in which objects are wielded by an anatomical component that is likely to be less well practiced, less dexterous, and less sensitive in perceiving such properties than the hands, the feet, or the torso - the head. Doing so provides a relatively strong test of both the task-specificity and the anatomical independence of perception of (proexteroceptive) properties of the person-object system by dynamic or effortful touch (cf. Wagman et al., 2017).

Experiment 1 investigates whether participants can perceive a proexteroceptive property of an object wielded by the head (i.e., the position of the head relative to the wielded object), and if so, whether such perception is supported by analogous task-specific sensitivity to $I$ (in particular, the product inertia $I_{\mathrm{yz}}$ ). Experiment 2 investigates the extent to which participants can differentiate between this property and a related exteroceptive property (i.e., partial length of a wielded object extending to one side of the head) under such circumstances.

\section{Experiment 1}

Previous experiments have shown (1) that people can perceive exteroceptive properties of an object wielded with the hand (e.g., whole or partial length of that object), (2) that these abilities generalize to objects wielded by other body parts (including the foot, the torso, and the head), and (3) that perception of such properties is supported by analogous sensitivities to exterospecific components of $I$ across anatomical components (Hajnal, Fonseca, Kinsella-Shaw, et al., 2007b; Palatinus et al., 2011; Wagman et al., 2017).

Previous research has also shown that when wielding an object by hand, people can perceive (exproprioceptive) relations between object and body (e.g., orientation of the object relative to the hand) as well as (proexteroceptive) relations between the body and object (e.g., the position of the hand relative to the object; see Pagano et al., 1996; Pagano et al., 1994). Experiment 1 investigates whether this ability generalizes to objects wielded by the head and, if so, whether this ability might be supported by analogous sensitivities to (proexteroceptive) components of $I$. Specifically, we investigated whether participants can perceive the position of the head relative to the wielded object.

We have two main hypotheses. First, we expected that (changes in) perceived proportional head position will reflect 
(changes in) actual proportional head position. Specifically, an analysis of variance will show an effect of actual proportional head position on perceived proportional head position. In addition, both at the level of the mean data and at the level of the individual participants, perceived proportional head position will be predicted by actual proportional head position. Specifically, simple regression will show that actual proportional head position accounts for a large and significant portion of the variance in proportional head position. Second, both at the level of the mean data and at the level of the individual participants, perceived head position will be predicted by a specific component of $I$ - in particular, the product of inertia, $I_{\mathrm{yz}}$ (see Pagano et al., 1996). Specifically, stepwise regression will select only $I_{\mathrm{yz}}$ (from among a number of components of $I$ ) which will account for a large and significant portion of the variance in perceived head position.

\section{Method}

\section{Participants}

Nine undergraduates from Illinois State University participated in this experiment in exchange for extra credit in psychology courses. Participants were deemed ineligible (prior to participation in the experiment) if their hair or headwear made it impossible for them to wear the helmet apparatus. Participants provided written informed consent prior to participation. The protocol was approved by the Illinois State University Institutional Review Board in accordance with the Declaration of Helsinki.

\section{Materials and apparatus}

Each participant stood in a designated area (approximately $35 \mathrm{~cm} \times 45 \mathrm{~cm}$ ) in front of a table-mounted report apparatus that consisted of an adjustable horizontal marker (a toy truck) along a 230-cm wooden track, at a height of $90 \mathrm{~cm}$, operated by a pulley system. Each participant wore a disposable shower cap and a plastic hard hat (ERB19762 Americana Cap Style, Woodstock, Georgia, USA) secured with an elastic chinstrap. A plastic cylinder $(5 \mathrm{~cm}$ outer diameter, $1.3 \mathrm{~cm}$ inner diameter, $7.5 \mathrm{~cm}$ length, $162 \mathrm{~g}$ mass) was attached to the back of the helmet so that it was parallel to the helmet brim (see Fig. 1). Two small rectangular pieces of cardboard were attached to each side of the helmet that occluded peripheral vision (of the rod), but allowed participants to see the report apparatus.

Participants wielded three different wooden rods $(65 \mathrm{~cm}$, $80 \mathrm{~cm}$, and $95 \mathrm{~cm}$; radius $=0.6 \mathrm{~cm}$ ) secured in the helmet such that a particular proportion of that $\operatorname{rod}(0.25,0.50$, or $0.75 \times$ the length of the rod, hereafter $0.25 \mathrm{~L}, 0.50 \mathrm{~L}$, and $0.75 \mathrm{~L}$ ) extended to the right of the (thumbscrew of the cylinder on the) helmet. A tape measure secured to the floor (not visible to the
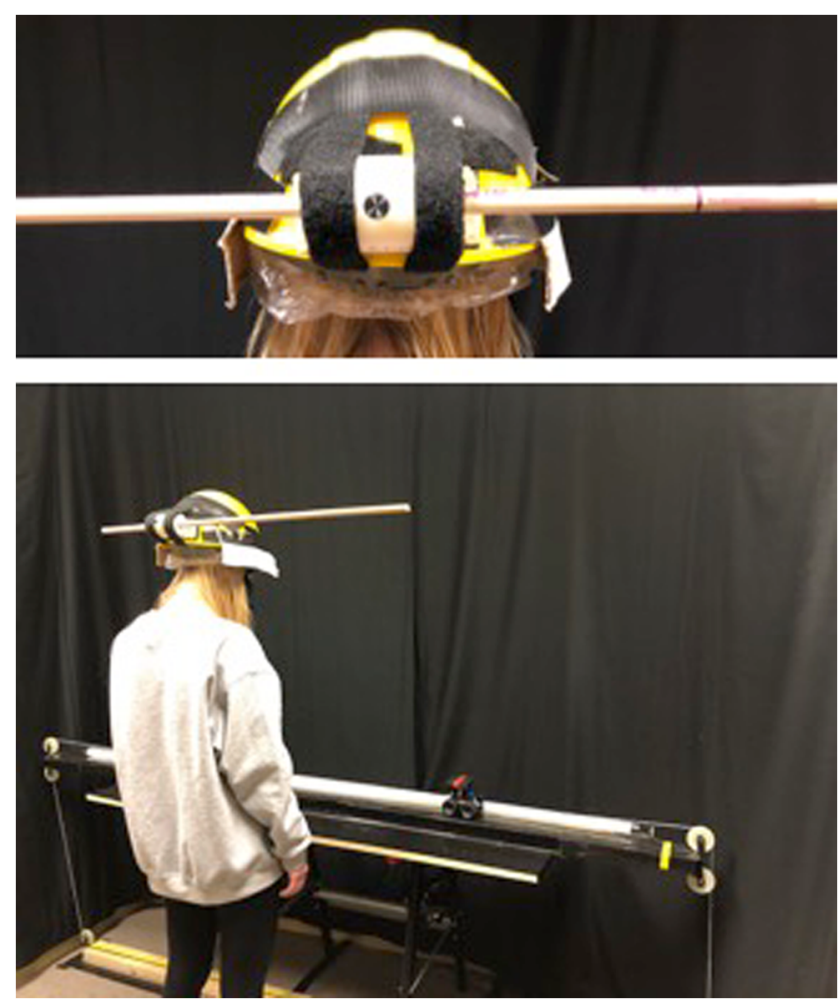

Fig. 1 The experimental apparatus and setup

participant) allowed the experimenter to read reports on each trial (see Fig. 1, Table 1).

\section{Procedure}

On a given trial, an experimenter inserted a rod into the plastic cylinder on the back of the helmet and secured it with a thumbscrew such that a proportion of the $\operatorname{rod}(0.25 \mathrm{~L}, 0.50 \mathrm{~L}$, or $0.75 \mathrm{~L})$ extended to the right of the (thumbscrew of the cylinder on the) helmet (i.e., to a particular proportional head position; see Table 1, Fig. 1). The experimenter then explained the task to the participant as follows: "Your job is to tell us what proportion of the rod is sticking out to each side. You will do this by adjusting the position of this truck so that the proportion of the track to the right of the truck corresponds to the proportion of the rod to your right and the proportion of the track to the left of the truck corresponds to the proportion of the rod to your left. For example, if it feels like a large portion (e.g., 90\%) of the rod is to your right and only a small portion (e.g., 10\%) is to your left, you might put the truck here (at a location such that $90 \%$ of the track is to the right of the marker and $10 \%$ of the track is to the left of the marker). If it feels like equal portions are on your right and your left, you might put the truck here (at location such that half of the track is on each side of the truck)." A second experimenter recorded the position of the marker (i.e., perceived proportional head position).

Wielding was unrestricted except that the participant was instructed not to step out of the designated area and to avoid 
Table 1 Properties of the experimental objects in Experiment 1

\begin{tabular}{cccccc}
\hline Rod length $(\mathrm{cm})$ & $\begin{array}{l}\text { Actual } \\
\text { head position } \\
\text { (proportion) }\end{array}$ & $\begin{array}{l}I_{\mathrm{xx}} \\
\left(\times 10^{4}\right) \\
\left(\mathrm{g} \times \mathrm{cm}^{2}\right)\end{array}$ & $\begin{array}{l}I_{\mathrm{zz}} \\
\left(\times 10^{4}\right) \\
\left(\mathrm{g} \times \mathrm{cm}^{2}\right)\end{array}$ & $\begin{array}{l}I_{\mathrm{yz}} \\
\left(\times 10^{4}\right) \\
\left(\mathrm{g} \times \mathrm{cm}^{2}\right)\end{array}$ \\
\hline 65 & 0.25 & 28.05 & 1.56 & 4.19 & $\begin{array}{l}\text { Perceived } \\
\text { head position } \\
(\mathrm{proportion})\end{array}$ \\
65 & 0.50 & 16.69 & 1.56 & 0.00 & 0.38 \\
65 & 0.75 & 28.05 & 1.56 & -4.19 & 0.51 \\
80 & 0.25 & 58.17 & 2.17 & 0.00 & 0.68 \\
80 & 0.50 & 34.17 & 2.17 & -7.20 & 0.33 \\
80 & 0.75 & 58.17 & 2.17 & 11.54 & 0.72 \\
95 & 0.25 & 109.53 & 2.93 & 0.00 & 0.26 \\
95 & 0.50 & 63.84 & 2.93 & -11.54 \\
\hline
\end{tabular}

Note. Inertial values were calculated about a rotation point in the neck by the following formulae (where $m=$ mass, $r=$ radius, $l=$ length, $: d_{\mathrm{CM}}=$ distance from midline of collar to center of mass of rod; $d_{\mathrm{C}}=$ distance from midline of collar attached to the helmet and the helmet brim, $6 \mathrm{~cm}$ for all participants):

$I_{\mathrm{xx}}=\left[(m / 12) \times\left(3 r^{2}+l^{2}\right)\right]+\left[\left(d_{\mathrm{CM}}^{2}+d_{\mathrm{C}}^{2}\right) \times m\right]$

$I_{\mathrm{zz}}=\left[(m / 2) \times\left(r^{2}\right)\right]+\left[\left(d_{\mathrm{C}}^{2}\right) \times m\right]$

$I_{\mathrm{yz}}=\left(m \times d_{\mathrm{CM}} \times d_{\mathrm{C}}\right)$

striking any object or surface with the rod. The participant was allowed to wield the rod as long as necessary and was allowed to continually adjust the marker until he or she was satisfied with the report. After the perceptual report was provided, an experimenter removed the rod from the plastic cylinder, the participant returned the marker to the zero point of the apparatus, and the same experimenter inserted and secured the next rod. The experimenter who was responsible for doing so stood on a stepstool behind the participant so as to perform this task as precisely and quickly as possible. Typically, the process of removing one rod and reinserting another rod took no more than $5 \mathrm{~s}$.

The participant was not provided any information about (the range or number of) possible lengths or proportions. Each participant wielded each rod at each proportion three times in a random order. Therefore, there was a total of 27 trials for each participant - three trials for each of the three rod lengths in each of the three rod proportion conditions. No practice trials were provided.

\section{Results}

Perceived head position on the rod was expressed as a proportion of the report rod's length extending to the right of the helmet, and this proportion was used as the measure of the subject's perception of head position on the wielded, occluded rod.

We conducted a 3 (proportional head position) $\times 3(\operatorname{rod}$ length) analysis of variance (ANOVA) on perceived proportion of the rod extending to the right of the helmet (i.e., on perceived proportional head position). There was a significant main effect of proportional head position, showing that perceived proportion of the rod increased as actual proportion increased, $F(2,16)=46.90, p<.05, \eta_{\mathrm{p}}{ }^{2}=0.85$. Follow-up $t$ tests with Bonferroni corrections found significant differences in perceived proportion across each of the three proportional head positions ( $0.75 \mathrm{~L}: M=0.70, S D=0.10>0.5 \mathrm{~L}: M=0.49$, $S D=0.06>0.25 \mathrm{~L}: M=0.32, S D=0.08$, all corrected $p s<.05$; see Fig. 2). There was also a main effect of Rod Length, $F(2,16)=5.32, p<.05, \eta_{\mathrm{p}}^{2}=0.40$, although follow-up $\mathrm{t}$ tests with Bonferroni corrections found no significant differences across rod lengths. Finally, there was an interaction of Proportional Head Position $\times$ Rod Length, $F(4,32)=2.89, p$ $<.05, \eta_{\mathrm{p}}{ }^{2}=0.26$. Follow-up $t$ tests with Bonferroni corrections found that the source of the interaction was that at the $0.25 \mathrm{~L}$ head position only, perceived proportion was longer for the 65 $\mathrm{cm} \operatorname{rod}(M=0.38, S D=0.08)$ than for the $95-\mathrm{cm} \operatorname{rod}(M=$

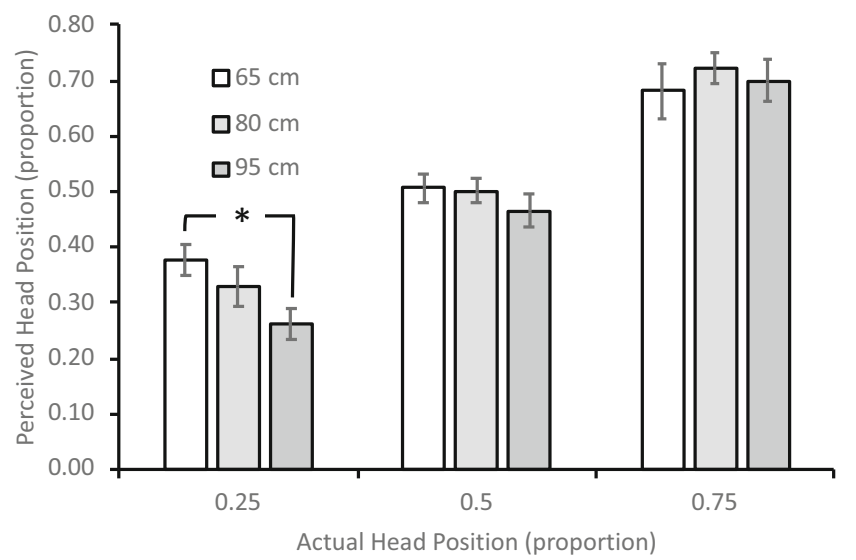

Fig. 2 Mean perceived head position in Experiment 1. Error bars indicate standard error of the mean. Asterisk indicates significant difference in follow up $t$ tests conducted to investigate the interaction of Proportional Head Position and Rod Length (corrected $p<.05$ ) 
$0.26, S D=0.03$ ), corrected $p<.05$ (see Fig. 2). Additional onesample $t$ tests with Bonferroni corrections found no differences between mean perceived proportion and actual proportion at $0.25 \mathrm{~L}, 0.50 \mathrm{~L}$, or $0.75 \mathrm{~L}$ (all corrected $p \mathrm{~s}>.016$ ).

At the level of the mean data, simple regression analysis showed that the proportional head position accounted for more than $95 \%$ of perceived proportional head position $\left(r^{2}=\right.$ .96 , slope $=0.75, p<.001$; see Fig. 3). At the level of the individual participant data, $r^{2}$ values ranged between .72 and .95 ( $p<.01$, for all participants).

At the level of the mean data, a stepwise regression was conducted to evaluate which inertial variables $\left(I_{\mathrm{xx}}, I_{\mathrm{zz}}\right.$, and $\left.I_{\mathrm{yz}}\right)$ predicted perceived proportional head position. At Step 1 of the analysis, $I_{\mathrm{yz}}$ entered into the regression equation and accounted for more than $90 \%$ of the variance in perceived proportional head position $\left(r^{2}=.92, p<.001\right)$. Neither $I_{\mathrm{xx}}(t=.93, p>.05)$ nor $I_{\mathrm{zz}}(t$ $=1.27, p>.05$ ) entered into the equation at Step 2 of the analysis. At the level of the individual participant data, $I_{\mathrm{yz}}$ accounted for between $69 \%$ and $94 \%$ of the variance in perceived proportional head position $(p<.01$, for all participants).

These results show that participants can perceive proportional head position on a wielded object. In particular, they completely supported the hypotheses that (1) (changes in) perceived proportional head position would reflect (changes in) actual proportional head position, and (2) perceived head position would be predicted by $I_{\mathrm{yz}}$.

\section{Experiment 2}

Experiment 1 found that people can perceive the position of the head relative to a wielded object. The ability to perceive the relative position of an effector on a wielded object generalized across anatomical components and is supported by sensitivity to the same component of $I$ (a particular product of inertia $\left.I_{\mathrm{yz}}\right)$ in each case. The results suggest that perception of proexteroceptive relations between body and a wielded object is anatomically independent. Experiment 2 investigates the

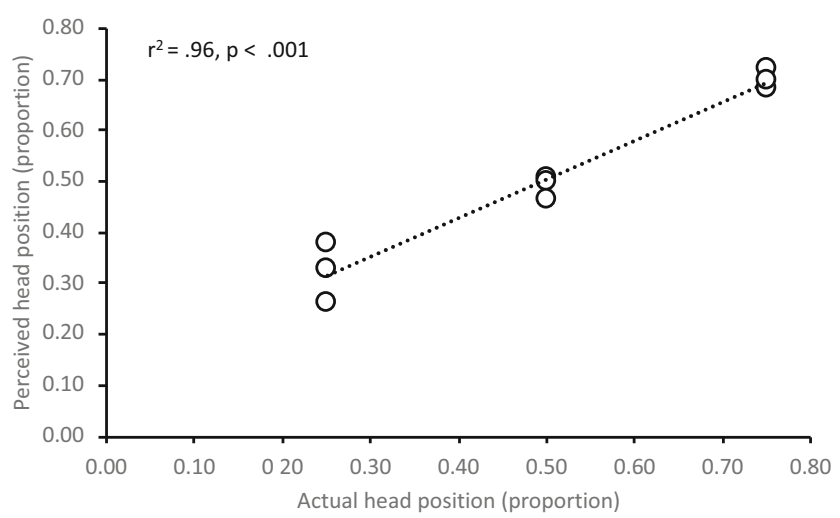

Fig. 3 Mean perceived head position as a linear function of actual head position extent to which perception of such relations is also task-specific. Specifically, Experiment 2 investigates the extent to which people can differentiate a proexteroceptive relation of the person-object system (relative position of the head on a wielded object) from a (related) exteroceptive property of the wielded object (partial length of the wielded object extending to one side of the head; see Pagano et al., 1996).

To investigate this hypothesis, participants reported perceived proportional head position and perceived partial length on two different sets of rods. Rod Set 1 consisted of an $80-\mathrm{cm}$ $\operatorname{rod}(\mathrm{RL}=80 \mathrm{~cm})$ and a $120-\mathrm{cm} \operatorname{rod}(\mathrm{RL}=120 \mathrm{~cm})$ secured into the helmet such that $0.50 \mathrm{~L}$ or $0.75 \mathrm{~L}$ extended to the right of the participant (see Fig. 5, top). Note that this combination of rod lengths and proportional head positions creates partial lengths of different magnitudes, but of the same proportion. Therefore, we expect perceived proportional head position to be affected by proportional head position, but not rod length. Conversely, we expect perceived partial length to be affected by both (actual) proportional head position and rod length. In other words, when proportional head position is held constant and partial length changes, participants ought to perceive changes in partial length but not proportional head position (see Fig. 6, top).

Rod Set 2 consisted of an $80-\mathrm{cm}$ rod and a $120-\mathrm{cm}$ rod secured into the helmet such that $60 \mathrm{~cm}$ or $40 \mathrm{~cm}$ of the rod extended to the right of the participant (see Fig. 5, bottom). Note that this creates partial lengths of the same magnitude, but of different proportions. Therefore, we expect an opposite pattern of results for this rod set. Specifically, we expect perceived proportional head position to be affected by both (actual) proportional head position and partial length. Conversely, we expect perceived partial length to be affected both by partial length, but not proportional head position. In other words, when partial length is held constant and proportional head position changes, participants ought to perceive changes in proportional head position, but not partial length (see Fig. 6).

Such a pattern of results across both sets of rods would show that participants can completely differentiate the relative position of the head on a wielded object from the partial length of the wielded object extending to one side of the head.

\section{Method \\ Participants}

Fourteen undergraduates from Illinois State University participated in this experiment in exchange for extra credit in psychology courses. As in Experiment 1, participants were deemed ineligible (prior to participation in the experiment) if their hair or headwear made it impossible to wear the helmet apparatus. Participants provided written consent prior to participation. The protocol was approved by the Illinois State 
University Institutional Review Board in accordance with the Declaration of Helsinki.

\section{Materials and apparatus}

The materials and apparatus were the same as in Experiment 1 , except that different rods were used. Rod Set 1 consisted of two rods $(\mathrm{RL}=80 \mathrm{~cm}$, and $\mathrm{RL}=120 \mathrm{~cm}$; radius $=0.6 \mathrm{~cm})$ secured in the helmet such that a particular proportion of the $\operatorname{rod}(0.50 \mathrm{~L}$ or $0.75 \mathrm{~L})$ extended to the right of the (thumbscrew of the cylinder on the) helmet. Rod Set 2 also consisted of two rods (again, $\mathrm{RL}=80 \mathrm{~cm}$ and $\mathrm{RL}=120 \mathrm{~cm}$; radius $=0.6 \mathrm{~cm}$ ), but secured in the helmet such that a particular portion of the length of that rod $(40 \mathrm{~cm}$ or $60 \mathrm{~cm}$ ) extended to the right of the (thumbscrew of the cylinder on the) helmet (see Fig. 5).

As in Experiment 1, the participant reported the perceived proportion of the rod extending to the right of the helmet by adjusting the location of the marker using a pulley system, such that the proportion of track extending to the right of the marker was the same as the proportion of rod extending to the right of the helmet. The participant reported perceived length of the portion of the rod extending to the right of the helmet by adjusting the location of the marker so that the distance between the marker and the zero point of the apparatus was the same length as this portion of the rod.

\section{Procedure}

On a given trial, an experimenter inserted a rod into the plastic cylinder on the back of the helmet and secured it with a thumbscrew such that either a particular proportion of that rod or a particular portion of the length of that rod extended to the right of the helmet (depending on rod set; see above). The participant wielded the rod with his or her neck and head and reported either the perceived proportion of the rod extending to the right of the helmet (i.e., perceived proportional head position) or the perceived partial length of the rod extending to the right of the helmet, as described above (depending on intention condition). The experimenter explained how to report perceived proportion using the same instructions as in Experiment 1. The experimenter explained how to report partial length as follows: "Your job is to tell us how long the portion of the rod sticking out to your right is. You will do this by adjusting the position of this truck so that the part of the rod that extends from the helmet to your right (and only this part of the rod) would exactly fit between truck and the (yellow) starting line. For example, if it feels like the portion of the rod sticking out to the right is very short (e.g., $10 \mathrm{~cm}$ ), you might place the truck here (so that the distance was approximately $10 \mathrm{~cm}$ ). If it feels like the portion of the rod sticking out to the right is very long (e.g., $75 \mathrm{~cm}$ ), you might place the truck here (so that the distance was approximately $75 \mathrm{~cm}$ )."
The same restrictions were placed on wielding as in Experiment 1. After the perceptual report was provided, an experimenter removed the rod from the plastic cylinder, and the participant returned the marker to the zero point of the apparatus.

Each participant completed both intention conditions (position and partial length) on both sets of rods. Intention conditions and rod sets were blocked and counterbalanced across participants. Each participant wielded all rods in a given set three times in a random order within each combination of intention condition and rod set.

As in Experiment 1, the participant was not provided any information about (range or number of) possible lengths or proportions. There was a total of 48 trials for each participant (four rods in a given set wielded three times in each of the two rod sets in each of the two intention conditions). No practice trials were provided.

\section{Results}

As in Experiment 1, perceived head position on the rod was expressed as a proportion of the report rod's length extending the right of the helmet, and this proportion was used as the measure of the subject's perception of head position on the wielded, occluded rod. Perceived partial length was expressed as a report (in $\mathrm{cm}$ ) of the rod's length extending to the right of the helmet.

Analysis of Rod Set 1

We conducted a 2 (Proportional head position) $\times 2(\operatorname{Rod}$ length) ANOVA on perceived proportion of the rod (i.e., on perceived proportional head position). As expected, there was a main effect of Proportional Head Position, $F(1,13)=13.87$, $p<.001, \eta_{\mathrm{p}}{ }^{2}=0.52$. Perceived proportion was larger when proportional head position was $0.75 \mathrm{~L}(M=0.63, S D=0.14)$ than when it was $0.50 \mathrm{~L}(M=0.47, S D=0.11)$. The main effect of Rod Length was not significant $(F<1)$. There was also an interaction of Proportional Head Position $\times$ Rod Length, $F(1$, 13 ) $=10.97, p<.001, \eta_{\mathrm{p}}{ }^{2}=0.46$ (see Fig. 7). Follow-up $t$ tests with Bonferroni corrections showed that at $0.50 \mathrm{~L}$ only, perceived proportion was larger for the $80-\mathrm{cm} \operatorname{rod}(M=0.52, S D$ $=0.13)$ than for the $120-\mathrm{cm} \operatorname{rod}(M=0.43, S D=0.11), t(13)=$ 3.35, corrected $p<.05$ (see Fig. 7, left; compare with Fig. 6, top left).

We conducted a parallel ANOVA on perceived length of the rod extending to the right (i.e., perceived partial length). There was a main effect of Rod Length, $F(1,13)=13.34, p<$. $001, \eta_{\mathrm{p}}{ }^{2}=0.51$. Perceived partial length was longer when rod length was $120 \mathrm{~cm}(M=58.3 \mathrm{~cm}, S D=24.7 \mathrm{~cm})$ than when it was $80 \mathrm{~cm}(M=49.0 \mathrm{~cm}, S D=21.3 \mathrm{~cm})$. There was a main effect of Proportional Head Position, $F(1,13)=6.29, p<.05$, $\eta_{\mathrm{p}}{ }^{2}=0.33$. Perceived partial length was larger when proportional head position was $0.75 \mathrm{~L}(M=60.2 \mathrm{~cm}, S D=29.0 \mathrm{~cm})$ 
than when it was $0.5 \mathrm{~L}(M=47.1 \mathrm{~cm}, S D=19.22 \mathrm{~cm})$. There was also an interaction of Proportional Head Position $\times$ Rod Length $F(1,13)=6.11, p<.05, \eta_{\mathrm{p}}{ }^{2}=0.33$ (see Fig. 7). Follow-up $t$ tests with Bonferroni corrections showed that at $0.75 \mathrm{~L}$ only, perceived length was longer for the $120-\mathrm{cm}$ rod $(M=67.7 \mathrm{~cm}, S D=31.4 \mathrm{~cm})$ than for the $80-\mathrm{cm} \operatorname{rod}(M=$ $52.6 \mathrm{~cm}, S D=27.4 \mathrm{~cm}), t(13)=5.54$, corrected $p<.05($ see Fig. 7, right; compare with Fig. 6, top right).

\section{Analysis of Rod Set 2}

We conducted a 2 (Partial Length) $\times 2$ (Rod Length) ANOVA on perceived proportion of the rod extending to the right of the helmet (i.e., on perceived proportional head position). There was a main effect of Rod Length, $F(1,13)=14.66, p<.01$, $\eta_{\mathrm{p}}{ }^{2}=0.53$. Perceived proportion was larger when rod length was $80 \mathrm{~cm}(M=0.51, S D=0.13)$ than when it was $120 \mathrm{~cm}(M=0.36$, $S D=0.09)$. There was also a main effect of Partial Length, $F(1$, $13)=14.61, p<.01, \eta_{\mathrm{p}}{ }^{2}=0.53$. Perceived partial length was longer when partial length was $60 \mathrm{~cm}(M=0.49, S D=0.11)$ than when it was $40 \mathrm{~cm}(M=0.38, S D=0.09)$. The interaction of Partial Length $\times$ Rod Length was not significant (see Fig. 8, left; compare with Fig. 6, bottom left).

We conducted a parallel ANOVA on perceived length of the rod extending to the right (i.e., perceived partial length). There was a main effect of Rod Length, $F(1,13)=6.51, p<.05, \eta_{\mathrm{p}}{ }^{2}=$ 0.33 . Perceived partial length was longer when rod length was $80 \mathrm{~cm}(M=46.5 \mathrm{~cm}, S D=23.5 \mathrm{~cm})$ than when it was $120 \mathrm{~cm}(M$ $=37.8 \mathrm{~cm}, S D=18.0 \mathrm{~cm})$. As expected, there was also a main effect of Partial Length, $F(1,13)=5.57, p<.05, \eta_{\mathrm{p}}{ }^{2}=0.30$. Perceived partial length was longer when partial length was $60 \mathrm{~cm}(M=46.2 \mathrm{~cm}, S D=20.4 \mathrm{~cm})$ than when it was $40 \mathrm{~cm}$ $(M=38.1 \mathrm{~cm}, S D=21.6 \mathrm{~cm})$. The interaction of Partial Length $\times$ Rod Length was marginally significant, $F(1,13)=4.08, p=.06$, $\eta_{\mathrm{p}}{ }^{2}=0.24$. Given the unexpected main effect of Rod Length, we conducted follow-up analyses on the marginal interaction. $T$ tests with Bonferroni corrections showed that the difference in perceived partial length occurred only for a partial length of $60 \mathrm{~cm}$ $t(13)=2.95$, corrected $p<.05$, but not for a partial length of $40 \mathrm{~cm}$ (see Fig. 8, right; compare with Fig. 6, bottom right).

For Rod Set 1, the combination of rod length and proportional head position created partial lengths of different magnitudes but of the same proportion. Reports of perceived proportional head position and partial length each partly reflected this pattern. When proportional head position was held constant and partial length varied, participants perceived changes in partial length but not proportional head position in two of the four cases (compare Fig. 7 with Fig. 6, top). In particular, in both cases, participants deviated from the expected pattern when equal portions of the rod extended to each side of the head (at $0.50 \mathrm{~L}$ ).

For Rod Set 2, the combination of rod length and partial length created partial lengths of different proportions but of the same magnitude. Reports of perceived proportional head position and partial length completely reflected this pattern; reports of partial length partly reflected this pattern (compare Fig. 8 with Fig. 6, bottom). When partial length was held constant and proportional head position varied, participants perceived changes in proportional head position but not partial length in three of the four cases. Participants deviated from the expected pattern only for reports of partial length, when a longer segment of the rod extend to the right (at $60 \mathrm{~cm}$ ).

\section{Regression analyses for combined rod sets}

We combined the data for the two rod sets and conducted a subset of the regression analyses conducted in Experiment 1. At the level of the mean data, simple regression analysis showed that (actual) proportional head position accounted for nearly $90 \%$ of the variance in perceived proportional head position $\left(r^{2}=.89\right.$, slope $\left.=0.61, p<.001\right)$. As in Experiment 1 , a stepwise regression was conducted to evaluate which inertial variables $\left(I_{\mathrm{xx}}, I_{\mathrm{zz}}\right.$, and $\left.I_{\mathrm{yz}}\right)$ predicted perceived proportional head position. At Step 1 of the analysis, $I_{\mathrm{yz}}$ entered into the regression equation and accounted for nearly $90 \%$ of the variance in perceived proportional head position $\left(r^{2}=.89, p<\right.$ $.001)$. Neither $I_{\mathrm{xx}}(t=1.63, p>.05)$ nor $I_{\mathrm{zz}}(t=2.45, p>.05)$ entered into the equation at Step 2 of the analysis.

At the level of the mean data, simple regression analysis showed that (actual) partial length accounted for nearly $80 \%$ of the variance in perceived partial length $\left(r^{2}=.78\right.$, slope $=$ $0.52, p<.01)$. A stepwise regression was conducted to evaluate which inertial variables $\left(I_{\mathrm{xx}}, I_{\mathrm{zz}}\right.$, and $\left.I_{\mathrm{yz}}\right)$ predicted perceived partial length. At Step 1 of the analysis, $I_{\mathrm{yz}}$ entered into the regression equation and accounted for more than $90 \%$ of the variance in perceived partial length $\left(r^{2}=.91, p<.001\right)$. Neither $I_{\mathrm{xx}}(t=1.73, p>.05)$ nor $I_{\mathrm{zz}}(t=1.36, p>.05)$ entered the equation at Step 2 of the analysis.

Overall, the pattern of results across both rod sets suggests that when wielding an object with the head, participants have at least some ability to differentiate a proexteroceptive relation of the person-object system (relative position of the head on a wielded object) from a (related) exteroceptive property of the wielded object (partial length of the wielded object extending to one side of the head). Moreover, the ability to perceive each property was supported (in part) by sensitivity to a component of $I$ (in this case, a particular product of inertia, $I_{\mathrm{yz}}$; but see Pagano et al., 1996).

\section{General discussion}

Previous research has found evidence of both task-specificity and anatomical independence in perception of properties of wielded objects. In particular, people can direct attention to different exteroceptive properties of wielded object (e.g., object length), and perception of such properties remains 
invariant across transformations including the anatomical component used to wield the object (see Hajnal, Fonseca, Harrison, et al., 2007a; Wagman et al., 2017). Such flexibility is grounded in sensitivity to task-specific (in this case, exterospecific) components of an invariant mechanical stimulation pattern - the rotational inertia $(I)$ of the person-object system. We investigated whether such task-specificity and anatomical independence generalize to perception of proexteroceptive properties of the person-object system (i.e., relative position of the body on a wielded object) when an object was wielded by the head.

Experiment 1 was directed at investigating the extent to which perception of proexteroceptive properties of the person-object system is anatomically independent. Pagano et al. (1996; Pagano et al., 1994) found that people can perceive relative position of the hand on a wielded object and that perception of this property was supported by detection of proexterospecific components of $I$ (in particular, the product inertia $I_{\mathrm{yz}}$ ). We investigated the extent to which people could perceive the relative position of the head on a wielded object and whether perception of this property was supported by detection of the same component of $I$. Participants reported perceived proportional head position on an object wielded by the head. Overall, we found that (changes in) perceived proportional head position reflected (changes in) actual proportional head position. Not only were there differences in mean perceived proportional head position at each of the three actual proportional head positions $(0.25 \mathrm{~L}, 0.50 \mathrm{~L}$, and $0.75 \mathrm{~L})$, but mean perceived proportional head position on the objects $(0.32,0.49$, and 0.70 , respectively) closely approximated actual head position (see Fig. 2). Moreover, at two of the three actual proportional head positions, changes in rod length did not (additionally) influence perceived proportional head position. The exception was at $0.25 \mathrm{~L}$. At this proportional head position only, perceived proportional head position decreased (and more closely approximated 0.25 ) as rod length increased. This pattern is comparable with one observed by Pagano et al. (1994; see their Table 1) for perception of perceived proportional hand position on a wielded object. Therefore, it may reflect a general psychophysical constraint on perception of this property more so than an anatomical constraint on perception of this property.

Consistent with this pattern of results, we also found that actual proportional head position accounted for almost all of the variance in mean perceived proportional head position (see Fig. 3). Moreover, $I_{\mathrm{yz}}$ (and not $I_{\mathrm{xx}}, I_{\mathrm{zz}}$ ) also accounted for almost all of the variance in mean perceived proportional head position (see Fig. 4), suggesting that perception of this property is supported by detection of proexterospecific components of $I$ (see Pagano et al., 1996; Pagano et al., 1994). Our conclusions on this point are somewhat tempered by the fact that we did not break the collinearity between actual proportional head position and $I_{\mathrm{yz}}$ (see Table 1). Pagano et al. (1996)

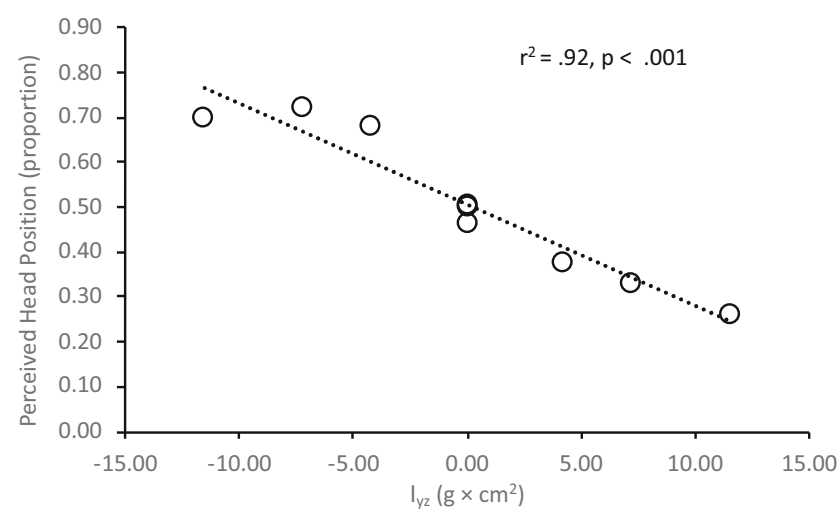

Fig. 4 Mean perceived head position as a linear function of $I_{\mathrm{yz}}$

found that perception of proportional hand position on a wielded object continued to be predicted by $I_{\mathrm{yz}}$ even when such collinearity was broken. A stronger test of this hypothesis with respect to perception of proportional head position is an important topic for future research. Nonetheless, together with previous research, such findings suggest perception by effortful touch is grounded in the intentional and anatomically independent detection of task-specific components of an invariant stimulation pattern (I) (Hajnal, Fonseca, Kinsella-Shaw, et al., 2007b; Pagano et al., 1996; Pagano et al., 1996; Palatinus et al., 2011).

Experiment 2 was primarily directed at investigating the extent to which perception of proexteroceptive properties of the person-object system is task-specific. That is, we investigated the degree to which the proexteroceptive property of relative position of the head on a wielded object could be differentiated from the related (but potentially independent) exteroceptive property of the partial length of a wielded object extending to one side of the head.

Participants reported perceived proportional head position and perceived partial length on two sets of objects. On one set of objects (Rod Set 1), the combination of rod lengths and proportional head positions was such that proportional head position was held constant, but partial length was not. Under such circumstances, we expected that participants would perceive changes in partial length, but not proportional head position. This pattern of results occurred in two of the four cases. In both cases, participants deviated from the expected pattern when equal portions of the rod extended to each side of the head (at $0.50 \mathrm{~L}$ ). At this head position, there was an unexpected difference in perceived head position and an unexpected nondifference in perceived partial length.

It is noteworthy, however, that despite the strict departure from the hypothesized results, there is still support for the overall hypothesis that participants could differentiate the two properties. In particular, while there was a main effect of proportional head position on perceived head position, the 
effect of rod length on perceived proportional head position was limited to an interaction. Therefore, on its own, proportional head position influenced perceived proportional head position (cf. Experiment 1), but rod length, on its own, did not. In addition, there are different patterns of results for perception of proportional head position and perception of partial length (compare Fig. 7, left and right). Although these differences are not quite those that we expected, they still show that perception of proportional head position differs from that of partial length.

On the other set of objects (Rod Set 2), the combination of rod lengths and partial lengths was such that partial length was held constant and proportional head position changed. Under such circumstances, we expected that participants would perceive changes in proportional head position, but not partial length (see Figs. 5 and 6). Reports of perceived proportional head position completely reflected this pattern. Reports of partial length only partly reflected this pattern. Participants deviated from the expected pattern only for reports of partial length when a longer segment of the rod extend to the right $($ at $60 \mathrm{~cm}$ ). At this partial length only, there was an unexpected difference in perceived partial length.

Again, it is noteworthy, that despite the strict departure from the hypothesized results, there is still support for the overall hypothesis that participants could differentiate the two properties. In particular, while there were main effects of both partial length and rod length on perceived partial length, follow-up tests showed that the main effect of rod length was driven by an interaction. Therefore, on its own, partial length influenced perceived partial length but, on its own, rod length did not.

Overall, although we did not support all hypotheses in all respects, the pattern of results across both rod sets suggests that when wielding an object with the head, participants have at least some ability to differentiate a proexteroceptive relation of the person-object system (relative position of the head on a wielded object) from a (related) exteroceptive property of the wielded object (partial length of the wielded object extending to one side of the head).

\section{Lawfully grounded flexibility and smart perceptual instruments}

Previous research has demonstrated lawfully grounded flexibility in perception of exteroceptive properties of an object wielded by hand, by foot, and by torso. Such research has shown that perception of such properties is both taskspecific and anatomically independent. The two experiments reported here build on and expand this body of work by investigating whether the same flexibility also occurs in perception of proexteroceptive properties of the person-object system (relative position of the head on a wielded object). Given that perceiving properties of an object attached to the head would seem to require the spontaneous assembly of a completely novel haptic perceptual instrument, the two experiments reported here served as a relatively strong test of the anatomical independence and task-specificity of perception by effortful or dynamic touch (cf. Wagman et al., 2017)

In short, the key experimental findings are that (1) the ability to perceive proexteroceptive properties of a person-object system generalizes across anatomical components, that (2) perception of such properties seems to be supported by analogous sensitivities to a proexterospecific component of $I$ across anatomical components (cf. Pagano et al., 1996), and (3) at least to some extent, the ability to differentiate proexteroceptive and exteroceptive properties of a personobject system also generalizes across anatomical components.

The results are consistent with the description of perception by effortful or dynamic touch as the coordination of anatomical components into a smart perceptual instrument - a measurement device that achieves a given perceptual goal by capitalizing on task specific, invariant stimulation patterns (Runeson, 1977; see Carello et al., 1992). Moreover, together with the results of previous studies, the results of the current study suggest that (1) smart perceptual instruments can be

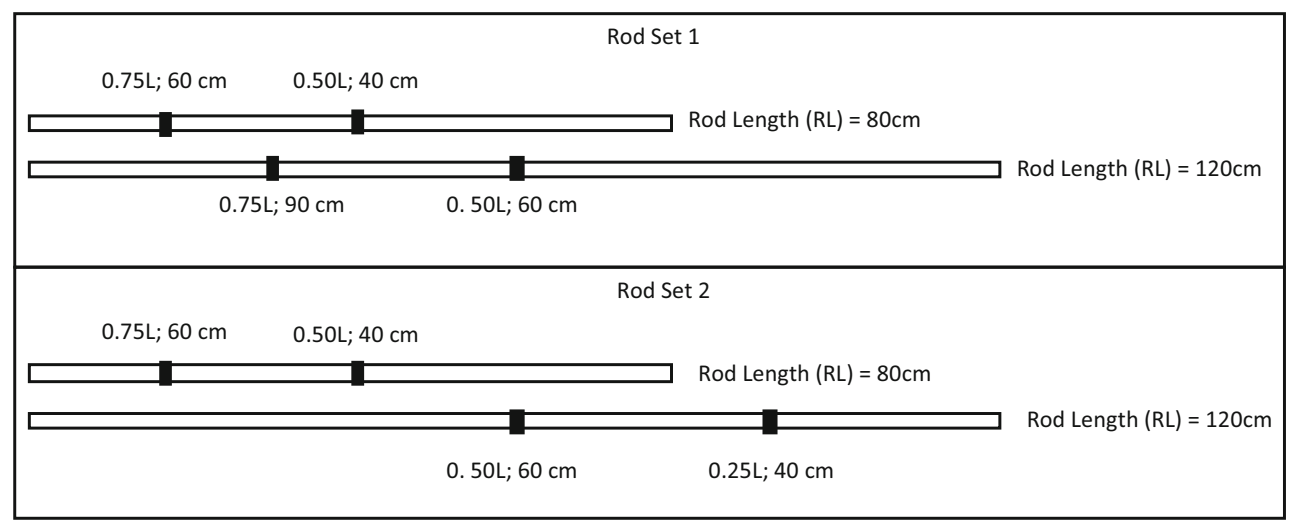

Fig. 5 Rod lengths, proportional head positions, and partial lengths on Rod Sets 1 and 2 
Rod Set 1: Head Position

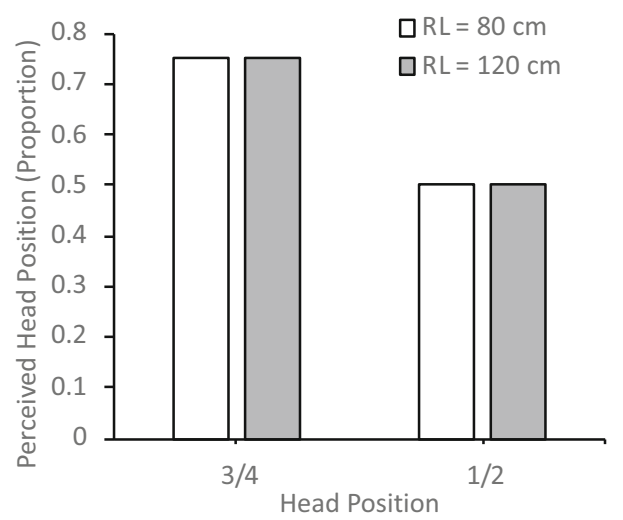

Rod Set 2 Head Position

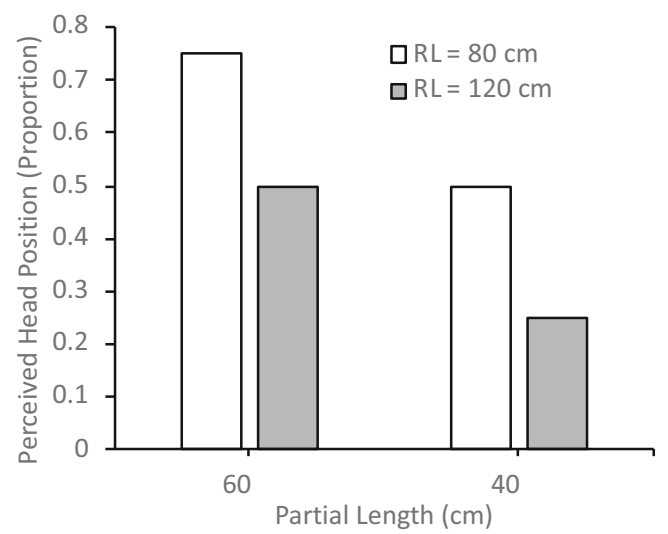

Rod Set 1: Partial Length

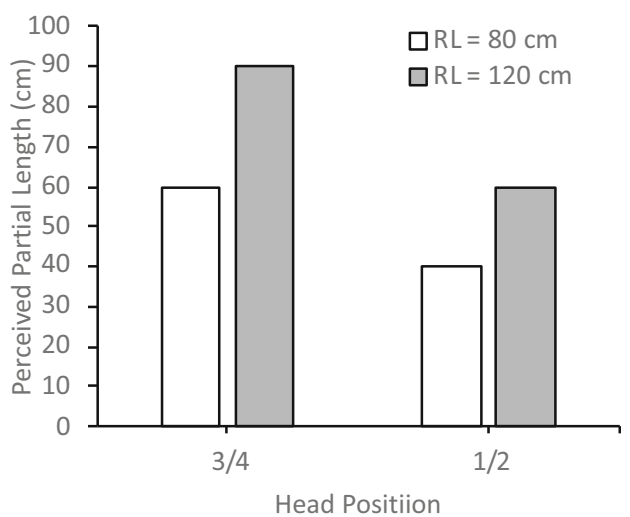

Rod Set 2: Partial Length

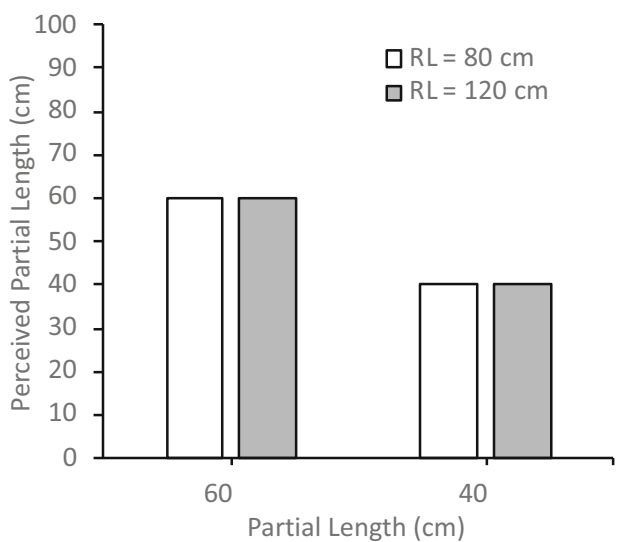

Fig. 6 Expected results for Experiment 2 for Rod Set 1 (top) and Rod Set 2 (bottom)

assembled across anatomical components and inert objects, (2) a given smart perceptual instrument can be assembled using different anatomical components, and (3) different smart perceptual instruments can be assembled using the same anatomical components (Wagman \& Hajnal, 2014a, 2014b; Wagman \& Hajnal 2016; Wagman et al., 2017).

\section{Rod Set 1: Head Position}

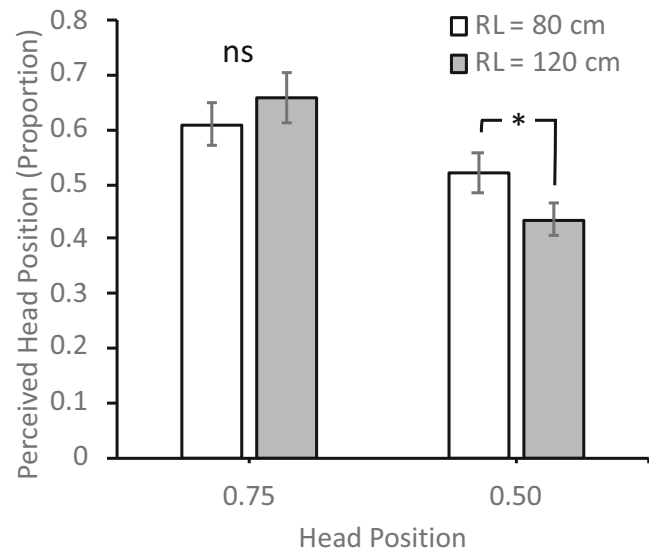

The information for effortful or dynamic touch and biotensegrity structures

To be informative to a perceiver, a particular stimulation pattern must be invariant across various transformations. In the context of perception by effortful or dynamic touch, exploring

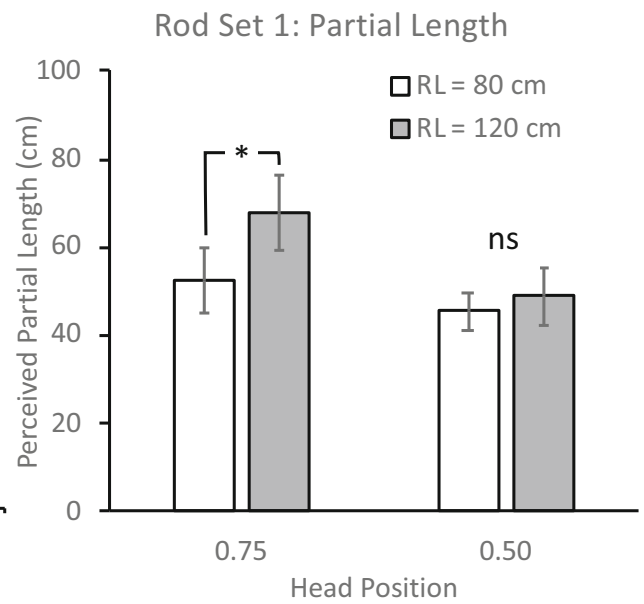

Fig. 7 Mean perceived head position and mean perceived partial length for Rod Set 1. Error bars indicate standard error of the mean. Asterisk indicates significant difference in follow up $\mathrm{t}$ tests $(\mathrm{p}<.05)$ 

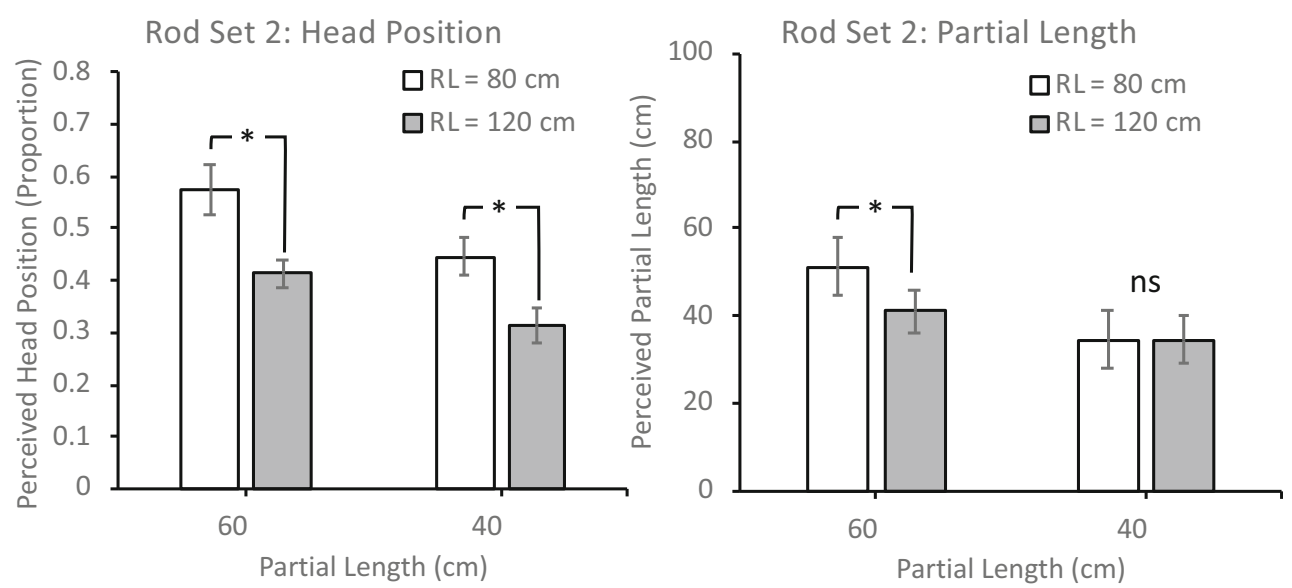

Fig. 8 Mean perceived head position and mean perceived partial length for Rod Set 2. Error bars indicate standard error of the mean. Asterisk indicates significant difference in follow up $t$ tests $(\mathrm{p}<.05)$

a wielded object lawfully generates patterns of deformation in muscular tissue that are (potentially) informative about exteroceptive, proprioceptive, exproprioceptive and proexteroceptive properties. The invariant nature of $I$ entails that sensitivity to (components of) $I$ support perception of properties of the person-object system over transformations, including wielding movements and trajectories, points of rotation, grasp types and locations, and anatomical components.

Moreover, the nature of the tissues that register such patterns may support the detection of such invariant patterns. The musculoskeletal system may best be described as a biotensegrity system - an intricately balanced mechanical structure supported by continuous tension elements and intermittent compression elements across all levels of scale (Turvey \& Fonseca, 2014). One of the key features of such a system is that local deformations are immediately redistributed across the entire system, creating a deformation field that is informative about the properties of the source of (but not necessarily the site of) the deformation. Therefore, all components of a biotensegrity system (e.g., the hand and the head) have potentially equivalent roles in registering and rebalancing applied forces, and all components of such as system can show analogous abilities to perceive exteroceptive, proprioceptive, exproprioceptive, and proexteroceptive properties of the person-object system. Both the task-specificity and anatomical independence exhibited in perception by effortful or dynamic touch may be generic characteristics of perceptual systems assembled out of tensegrity structures embedded in lawfully structured energy fields (Cabe, 2018; Turvey, 2019; Wagman \& Hajnal, 2014b).

Acknowledgements We thank Gabriela Wec, Sarah Caputo, Ellen Bjerga, and Katie Kresse for help with data collection.

Open practices statement The data and materials for all experiments are available upon request, and neither of the experiments was preregistered

\section{References}

Burton, G., \& Turvey, M. T. (1990). Perceiving the lengths of rods that are held but not wielded. Ecological Psychology, 2(4), 295-324.

Cabe, P. A. (2018). All perception engages the tensegrity-based haptic medium. Ecological Psychology. doi:https://doi.org/10.1080/ 10407413.2018.1526037

Carello, C., Fitzpatrick, P., Domaniewicz, I., Chan, T., \& Turvey, M. T. (1992). Effortful touch with minimal movement. Journal of Experimental Psychology: Human Perception and Performance, 18, 290-302.

Carello, C., \& Turvey, M. T. (2015) Dynamic (effortful) touch. Scholarpedia, 10(4), 8242.

Carello, C., \& Turvey, M. T. (2017). Useful dimensions of haptic perception: 50 years after the senses considered as perceptual systems. Ecological Psychology, 29, 95-121.

Fitzpatrick, P., Carello, C., \& Turvey, M. T. (1994). Eigenvalues of the inertia tensor and exteroception by the muscular sense." Neuroscience, 60(2), 551-568.

Gibson, J. J. (1966). The senses considered as perceptual systems. Boston, MA: Houghton Mifflin.

Hajnal, A., Fonseca, S., Harrison, S., Kinsella-Shaw, J., \& Carello, C. (2007a). Comparison of dynamic (effortful) touch by hand and foot. Journal of Motor Behavior, 39, 82-88.

Hajnal, A., Fonseca, S., Kinsella-Shaw, J., Da Silva, P., Carello, C., \& Turvey, M. T. (2007b). Comparison of dynamic (effortful) touch by hand and foot: Perception of partial length. Neuroscience Letters, 419, 5-9.

Lee, D. N. (1978). The functions of vision. In H. L. Pick \& E. Saltzman (Eds.), Modes of perceiving and processing information (pp. 159170). Hillsdale, NJ: Erlbaum.

Pagano, C. C., Carello, C., \& Turvey, M. T. (1996). Exteroception and exproprioception by dynamic touch are different functions of the inertia tensor. Perception \& Psychophysics, 58, 1191-1202.

Pagano, C. C., Fitzpatrick, P., \& Turvey, M. T. (1993). Tensorial basis to the constancy of perceived object extent over variations of dynamic touch. Perception \& Psychophysics, 54(1), 43-54.

Pagano, C. C., Kinsella-Shaw, J., Cassidy, P., \& Turvey, M. T. (1994). Role of the inertia tensor in perceiving where an object is grasped. Journal of Experimental Psychology: Human Perception and Performance, 20, 276-285.

Palatinus, Z., Carello, C., \& Turvey, M. T. (2011). Principles of partwhole selective perception extend to the torso. Journal of Motor Behavior, 43, 87-93 
Runeson, S. (1977). On the possibility of "smart" perceptual mechanisms. Scandinavian Journal of Psychology, 18, 172-179.

Shaw, R. (2001). Processes, acts, and experiences: Three stances on the problem of intentionality. Ecological Psychology, 13, 275-314.

Turvey, M. T. (2019). Lectures on perception: An ecological perspective. New York, NY: Routledge.

Turvey, M. T., Burton, G., Amazeen, E. L., Butwill, M., \& Carello, C. (1998). Perceiving the width and height of a hand-held object by dynamic touch. Journal of Experimental Psychology: Human Perception and Performance, 24(1), 35.

Turvey, M. T., \& Fonseca, S. T. (2014). The medium of haptic perception: A tensegrity hypothesis. Journal of Motor Behavior, 46(3), 143-187.

Wagman, J. B., \& Hajnal, A. (2014a). Getting off on the right (or left) foot: perceiving by means of a rod attached to the preferred or nonpreferred foot. Experimental Brain Research, 232, 3591-3599.

Wagman, J. B., \& Hajnal, A. (2014b). Task-specificity and anatomical independence in perception of surface properties by means of a wielded object. Journal of Experimental Psychology: Human Perception \& Performance, 40, 2372-2391.

Wagman, J. B., \& Hajnal, A. (2016). Use your head! Perception of action possibilities by means of an object attached to the head. Experimental Brain Research, 234, 829-836.

Wagman, J. B., Langley, M. D., \& Higuchi, T. (2017). Turning perception on its head: Cephalic perception of whole and partial length of a wielded object. Experimental Brain Research, 235, 153-167.

Wagman, J. B., \& Mason, J. M. (2019). Selective perception in probing by foot: Perceiving the length of a probe and the distance of a surface probed. Manuscript submitted for publication.

Publisher's note Springer Nature remains neutral with regard to jurisdictional claims in published maps and institutional affiliations. 